\title{
Die Therapie der idiopathischen Lungenfibrose - was bleibt?
}

\author{
Therapy of Idiopathic Pulmonary Fibrosis - What Remains?
}

Die idiopathische Lungenfibrose (IPF) wird heute als langsam progrediente, meist tödlich verlaufende Lungenerkrankung verstanden, die sich primär durch eine Fibroblastenproliferation in der Folge einer Schädigung des Alveolarepithels auszeichnet. Konsekutiv kommt es zum Umbau der extrazellulären Matrix und zur irreversiblen Zerstörung der Lungenarchitektur. Der IPF wird heute ausschließlich das histopathologische Muster der usual interstitial pneumonia (UIP) zugeordnet [1 - 3]. Ein zentrales Merkmal dieses Musters ist die Bildung von multiplen fleckförmigen Fibroblastenherden (so genannten fibroblastären Foki), welche offenbar den Stellen entsprechen, an denen die aktive Kollagensynthese stattfindet. Entzündliche Veränderungen sind vermutlich sekundärer Natur. Das jahrelang gültige Konzept, dass sich aus einer initialen Alveolitis (Infiltration aus Entzündungszellen) die Fibrose entwickelt, wird heute durch die Vorstellung abgelöst, dass die IPF eine primäre epithelial/fibroblastäre Erkrankung ist [4].

Vor dem Hintergrund dieses neuen pathogenetischen Konzeptes verstehen wir das therapeutische Dilemma besser: Mit immunsuppressiver Therapie sind nur die sekundären entzündlichen Läsionen zu beeinflussen. Auf eine Prednisontherapie in Kombination mit Azathioprin oder Cyclophosphamid sprechen nur maximal 30\% der Patienten vorübergehend an. Für die Hemmung der Fibroblastenaktivität, des Primärereignisses, stehen noch keine wirksamen zugelassenen Medikamente zur Verfügung.

Vor 5 Jahren machte ein Artikel im New England Journal of Medicine Furore. Ziesche u. Mitarb. berichteten über sagenhaft anmutende Effekte von Interferon(IFN)- $\gamma$-1b bei Patienten mit IPF
[5]. 9 Patienten, die 12 Monate lang mit IFN- $\gamma-1 \mathrm{~b}$ in Kombination mit einer niedrigen Prednisolondosis behandelt wurden, zeigten alle eine Verbesserung in der Lungenfunktion, während die 9 Patienten in der nur mit Prednisolon behandelten Gruppe sich alle mit der Lungenfunktion verschlechterten. Die Studie war zwar randomisiert, aber nicht Placebo-kontrolliert. Diese Studie wurde einerseits kritisch bewertet, und eine später vorgenommene Analyse zeigte, dass nicht alle Patienten eine IPF nach der neuen Definition hatten, war andererseits aber der Anlass, dass immer mehr Patienten, vor allem in den USA, im „off-label use“ mit dieser Substanz behandelt wurden.

Im letzten Jahr wurden erstmals kleine Fallserien publiziert, welche die günstigen Erfahrungen von Ziesche u. Mitarb. mit IFN- $\gamma-1 \mathrm{~b}$ nicht bestätigen konnten [6, 7]. Es handelte sich um Patienten mit fortgeschritteneren Krankheitsstadien. Prasse u. Mitarb. behandelten 5 Patienten mit IPF in ähnlicher Weise wie Ziesche u. Mitarb. Nur 1 Patient zeigte eine Verbesserung in der Lungenfunktion, 4 Patienten verschlechterten sich, die Totalkapazität lag bei diesen Patienten im Mittel bei $57 \%$, während sie bei Ziesche u. Mitarb. 70\% betrug [6]. Französische Autoren berichteten, dass 4 Patienten im Endstadium der IPF (Totalkapazität unter $45 \%$ oder CO-Diffusionskapazität unter 30\%) ein ARDSartiges Bild in engem Bezug zum Beginn der IFN- $\gamma$-1b-Therapie entwickelten und schließlich daran starben [7].

Die Resultate einer kürzlich publizierten Phase-III-Studie mit IFN- $\gamma$ bei IPF wurden daher mit großer Hoffnung erwartet, doch leider konnte auch diese Studie die Frage nicht endgültig beantworten, ob INF- $\gamma$ bei IPF wirksam ist [8]. In dieser doppelblinden,

Institutsangaben

Anmerkung

Prof. Detlef Kirsten zum 60. Geburtstag in freundschaftlicher Verbundenheit 
multinationalen Studie wurden 330 Patienten mit IPF, die nicht auf eine Behandlung mit Corticosteroiden ansprachen, für eine Therapie mit IFN- $\gamma-1 b 200 \mu \mathrm{g} 3 \times$ wöchentlich oder für Placebo randomisiert. Über einen Zeitraum von 58 Wochen hinweg wurde eine signifikante Wirkung auf den primären Endpunkt, nämlich die Zeitdauer bis zur Krankheitsprogression, definiert als Verschlechterung von Lungenfunktionsgrößen oder Tod, nicht erzielt. Es fand sich auch kein signifikanter Mortalitätsunterschied (sekundärer Endpunkt) im Gesamtkollektiv (Mortalität $10 \%$ in der Interferon-Gruppe, $17 \%$ in der Placebo-Gruppe, $p=0,08$ ). Allerdings fand sich in einer exploratorischen post-hocAnalyse unter INF- $\gamma$-Therapie eine signifikante Abnahme der Mortalität bei einer Subgruppe von Patienten mit relativ guter Lungenfunktion. Diese große randomisierte Studie konnte also die hervorragenden Resultate von Ziesche u. Mitarb. nicht bestätigen. Möglicherweise ist IFN- $\gamma$ nur bei Patienten im frühen bis mittleren Krankheitsstadium wirksam. Eine Studie zur Beantwortung dieser Frage beginnt zur Zeit in Nordamerika und Europa. In diese doppelblinde, Placebo-kontrollierte Phase-III-Studie werden ausschließlich Patienten im frühen bis mittleren Krankheitsstadium (definiert aufgrund der Lungenfunktion und der Zeitdauer seit Diagnose) aufgenommen. Der primäre Endpunkt ist die Überlebenszeit. 600 Patienten in etwa 70 Studienzentren werden im Verhältnis 2:1 randomisiert, die Behandlungsdauer wird mindestens 24 Monate betragen.

Welche anderen Optionen können in der Therapie der IPF in Zukunft erwartet werden? N-Acetylcystein (NAC) wirkt als Antioxidans. In einer Phase-II-Studie bei einem heterogenen Patientenkollektiv mit Lungenfibrosen zeigte sich nach 3 Monaten ein Trend zur Lungenfunktionsverbesserung [9]. Auf dem diesjährigen ERS-Kongress werden die mit Spannung erwarteten endgültigen Resultate der großen europäischen, multizentrischen Phase-III-Studie mit NAC, hochdosiert ( $3 \times 600 \mathrm{mg}$ täglich) zusätzlich zur Standardtherapie Prednison/Azathioprin gegeben, vorgestellt (IFIGENIA-Studie). Die vorläufige Analyse der Daten, von Jürgen Behr bereits auf dem DGP-Kongress im März dieses Jahres vorgetragen, sieht vielversprechend aus. Der Endothelinrezeptorantagonist Bosentan und der TNF- $\alpha$-Antagonist Etanercept werden zur Zeit in doppelblinden Placebo-kontrollierten Studien geprüft. Pirfenidon ist eine weitere interessante antifibrotische Substanz. In Japan wurde kürzlich eine Phase-III-Studie vorzeitig abgebrochen, da in der Placebo-Gruppe deutlich mehr akute Exazerbationen der IPF beobachtet wurden. Weitere klinische Studien mit Pirfenidon sind in der Planung.

Aus zellbiologischen und tierexperimentellen Untersuchungen sind andere antifibrotische Substanzen bekannt, die vielleicht in der Therapie der IPF Eingang finden werden [4]. Dazu zählen Captopril (hemmt die Apoptose humaner Alveolarepithelien und die Fibroblastenproliferation), Lovastatin (hemmt die Bildung von Granulationsgewebe und induziert die Apoptose von Fibroblasten), Relaxin (inhibiert die Kollagensynthese) und Prostaglandin E2 (hemmt die Fibroblastenproliferation).

Zusammengefasst finden sich heute zahlreiche antifibrotisch wirksame Moleküle in der Pipeline. Aussagekräftige Phase-IIIStudien, welche stringente diagnostische Kriterien entsprechend des neuen Konsensusstatements [3] und relevante und standardisierte Endpunkte berücksichtigen, sind bei IPF heute möglich. Vielleicht wird in Zukunft eine multi-medikamentöse Behandlung verfügbar sein, die aus einer antifibrotischen Substanz zur Hemmung der Fibroblastenproliferation besteht, einer Immunsuppression, um die sekundäre Inflammation zu beeinflussen, und einem Antioxidans, um vor den oxidativen Mechanismen zu schützen. Es ist zu hoffen, dass mit diesem Ansatz die IPF eine besser behandelbare Erkrankung wird.

\section{Literatur}

${ }^{1}$ Bjoraker JA, Ryu JH, Edwin MK et al. Prognostic significance of histopathologic subsets in idiopathic pulmonary fibrosis. Am J Respir Crit Care Med 1998; 157: 199-203

2 Flaherty KR, Toews GB, Travis WD et al. Clinical significance of histological classification of idiopathic interstitial pneumonia. Eur Respir J 2002; 19: $275-283$

3 Travis WD, King TE, Bateman ED et al. ATS/ERS international multidisciplinary consensus classification of idiopathic interstitial pneumonias. General principles and recommendations. Am J Respir Crit Care Med 2002; 165: 277-304

${ }^{4}$ Selman M, King Jr TE, Pardo A. Idiopathic pulmonary fibrosis: prevailing and evolving hypotheses about its pathogenesis and implication for therapy. Ann Intern Med 2001; 134: 136 - 151

${ }^{5}$ Ziesche R, Hofbauer E, Wittmann K et al. A preliminary study of longterm treatment with interferon- $\gamma-1 \mathrm{~b}$ and low-dose prednisolone in patients with idiopathic pulmonary fibrosis. N Engl J Med 1999; 341: $1264-1269$

${ }^{6}$ Prasse A, Müller KM, Kurz C et al. Does interferon- $\gamma$ improve pulmonary function in idiopathic pulmonary fibrosis? Eur Respir J 2003; 22: 906-911

${ }^{7}$ Honoré I, Nunes $\mathrm{H}$, Groussard $\mathrm{O}$ et al. Acute respiratory failure after interferon- $\gamma$-therapy of end-stage pulmonary fibrosis. Am J Respir Crit Care Med 2003; 167: 953-957

${ }^{8}$ Raghu G, Brown KK, Bradford WZ et al. A placebo-controlled trial of interferon- $\gamma$-Ib in patients with idiopathic pulmonary fibrosis. N Engl J Med 2004; 350: 125 - 133

${ }^{9}$ Behr J, Mayer K, Degenkolb B et al. Antioxidative and clinical effects of high-dose N-acetylcysteine in fibrosing alveolitis. Am J Respir Crit Care Med 1997; 156: 1897-1901 